\title{
Mycobacterium abscessus D-alanyl-D-alanine dipeptidase induces the maturation of dendritic cells and promotes Th1-biased immunity
}

\author{
Seung Jun Lee ${ }^{1, \#}$, Jong-Hwa Jang ${ }^{2, \#}$, Gun Young Yoon ${ }^{1}$, Da Rae Kang ${ }^{1}$, Hee Jo Park ${ }^{1}$, Sung Jae Shin ${ }^{3}$, Hee Dong Han ${ }^{1}$, \\ Tae Heung Kang ${ }^{1}$, Won Sun Park ${ }^{4}$, Young Kyung Yoon ${ }^{5}$, Byoung Yul Soh ${ }^{6}$, In Duk Jung ${ }^{1, *}$ E Yeong-Min Park ${ }^{9, *}$ \\ ${ }^{1}$ Department of Immunology, Laboratory of Dendritic Cell Differentiation and Regulation, School of Medicine, Konkuk University, \\ Chungju 27478, ${ }^{2}$ Department of Dental Hygiene, Hanseo University, Seosan 31962, ${ }^{3}$ Department of Microbiology, Institute for \\ Immunology and Immunological Diseases, Brain Korea 21 PLUS Project for Medical Science, Yonsei University College of Medicine, Seoul \\ 03722, ${ }^{4}$ Department of Physiology, School of Medicine, Kangwon National University, Chuncheon 24341, ${ }^{5}$ Division of Infectious Diseases, \\ Department of Internal Medicine, Korea University Anam Hospital, College of Medicine, Korea University, Seoul 02841, ${ }^{6}$ Department of \\ Biochemistry, College of Medicine, Seonam University, Namwon 55724, Korea
}

\begin{abstract}
Mycobacterium abscessus, a member of the group of non-tuberculous mycobacteria, has been identified as an emerging pulmonary pathogen in humans. However, little is known about the protective immune response of antigenpresenting cells, such as dendritic cells (DCs), which guard against $M$. abscessus infection. The $M$. abscessus gene $M A B 1843$ encodes D-alanyl-D-alanine dipeptidase, which catalyzes the hydrolysis of D-alanyl-D-alanine dipeptide. We investigated whether MAB1843 is able to interact with DCs to enhance the effectiveness of the host's immune response. MAB1843 was found to induce DC maturation via toll-like receptor 4 and its downstream signaling pathways, such as the mitogen-activated protein kinase and nuclear factor kappa B pathways. In addition, MAB1843-treated DCs stimulated the proliferation of $\mathrm{T}$ cells and promoted Th1 polarization. Our results indicate that MAB1843 could potentially regulate the immune response to $M$. abscessus, making it important in the development of an effective vaccine against this mycobacterium. [BMB Reports 2016; 49(10): 554-559]
\end{abstract}

${ }^{*}$ Corresponding authors. In Duk Jung, Tel: +82-2-2049-6330; Fax +82-2-2049-6192; E-mail: jungid@kku.ac.kr, Yeong-Min Park, Tel: +82-2-2049-6330; Fax: +82-2-2049-6192; E-mail: immun3023@ kku.ac.kr

${ }^{\#}$ These authors contributed equally to this work.

https://doi.org/10.5483/BMBRep.2016.49.10.080

Received 10 May 2016, Revised 30 May 2016, Accepted 19 July 2016

Keywords: Dendritic cells, MAB1843, MAPK, Mycobacterium abscessus, Thl polarization

\section{INTRODUCTION}

Mycobacterium abscessus is the most well known rapidly growing mycobacterium (RGM), existing in natural habitats such as soil and water $(1,2)$ and usually found in intracellular compartments within an infected host. Infection by this mycobacterium can occur in various parts of the host's body, regardless of their immunity. Approximately $80 \%$ of pulmonary RGM infections in the USA are attributed to $M$. abscessus (3). Unfortunately, the resistance of this species to antituberculosis drugs and antibiotics has become a major obstacle to its treatment in infected patients (4-6).

Recent studies have revealed that antigen-presenting cell (APC)-mediated innate and adaptive immunities play a pivotal role in combating M. abscessus infection (7-9). These studies also pointed out the importance of research on the pathogenicity of $M$. abscessus and relevant immunotherapies. In the $M$. abscessus-infected mouse model, the interferongamma (IFN- $\gamma$ ) and tumor necrosis factor alpha (TNF- $\alpha$ ) secreted by various immune cells play a key role as regulators of the bacterial infection $(10,11)$. Furthermore, M. abscessus induces dendritic cell (DC) and macrophage secretion of TNF- $\alpha$, interleukin-6 (IL-6), and IL-12p70 via the toll-like receptor 2 (TLR2) and TLR4 pathways $(7,8)$.

DCs are well known as the most powerful APCs. In peripheral tissues, immature DCs express low levels of costimulatory and major histocompatibility complex (MHC) molecules and exhibit a high level of endocytosis $(12,13)$. When DCs recognize pathogens via pattern recognition receptors, they start maturing, and in the process express high levels of costimulatory and MHC molecules, and proinflammatory cytokines, and their endocytosis capacity is downregulated. In addition, DC maturation occurs as the cells migrate towards the lymph nodes where they present antigens to naïve $\mathrm{T}$ cells, which induces $\mathrm{T}$ cell activation and 
proliferation. In this way, DCs act as the link between innate immunity and adaptive immunity, and hence, they have gained much attention in cancer immunotherapy research (12-15).

Mycobacterial proteins, such as PE-RGRS, LprA, MAB2560, Rv0652, Rv0462, MAP1305, HspX, and HBHA, have been shown to induce DC maturation and T cell activation (8, 16-22). These proteins activate TLR2 or TLR4 signaling and can be used as adjuvants for DC maturation. We recently suggested MAB2560 as the first ligand isolated from $M$. abscessus that induces the activation of DCs via TLR4; however, little is known about how M. abscessus-derived ligands affect the immune system.

MAB1843, a 222-amino-acid D-alanyl-D-alanine dipeptidase (D-Ala-D-Ala dipeptidase; EC number: 3.4.13.22), is a hypothetical antigen of $M$. abscessus. However, the function of MAB1843 in M. abscessus and the manner in which it affects the immune system remain unknown.

In this study, we aimed to elucidate the effects of MAB1843 on the immune system, with a focus on the maturation of DCs as well as their endocytosis and cytokine-producing abilities. In particular, we examined whether MAB1843 affects DC maturation through TLRs and how the activated DCs affect T cells. Knowledge on these mechanisms could contribute to the development of vaccines against $M$. abscessus.
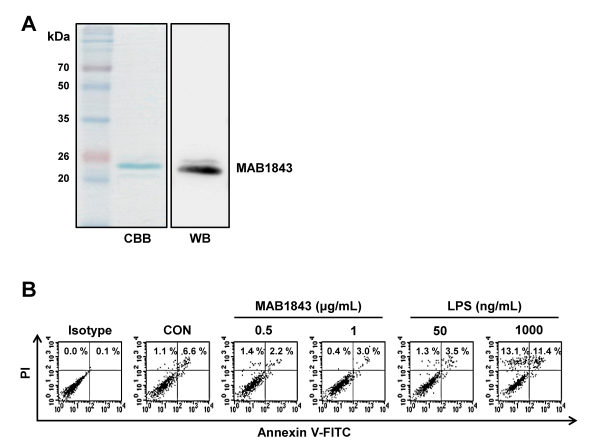

C

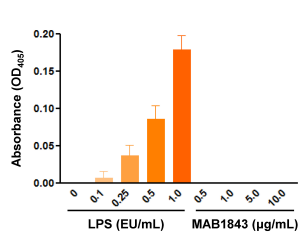

Fig. 1. Purification and cytotoxicity of recombinant MAB1843. (A) Recombinant MAB1843 was purified by affinity chromatography with Ni-NTA resin and then subjected to SDS-PAGE and staining with Coomassie Brilliant Blue (left panel), as well as analysis by western blotting using 1:1000 mouse anti-His tag antibodies (right panel). (B) Dendritic cells were treated with the indicated concentrations of MAB1843 and LPS for $24 \mathrm{~h}$ and then stained with Annexin V/PI. The results are representative of three independent experiments. (C) Endotoxin levels in the purified MAB1843 were analyzed by the LAL endotoxin assay kit.

\section{RESULTS}

\section{MAB1843 induces dendritic cell maturation}

First, we inspected the purity and toxicity of the recombinant MAB1843 before testing its physiological effect on DCs. The purity of MAB1843 was confirmed by Coomassie Brilliant Blue staining and western blot analysis using anti-His antibody (Ab). The apparent molecular size of the purified protein was approximately $24 \mathrm{kDa}$ (Fig. 1A). In addition, the toxicity of MAB1843 on DCs was tested using Annexin V/propidium iodide (PI) staining and analyzed by fluorescence-activated cell sorting. Compared with the effect of a high dose of lipopolysaccharide (LPS; $1,000 \mathrm{ng} / \mathrm{ml}$ ) as a positive control, MAB1843 was not cytotoxic to DCs (Fig. 1B). To exclude the effects of endotoxin contamination, we also checked for endotoxins in the purified MAB1843. Using the limulus amebocyte lysate (LAL) endotoxin assay kit (Lonza, Basel, Switzerland), we found the endotoxin levels in the purified MAB1843 to be $<0.1 \mathrm{EU} / \mathrm{ml}$ (Fig. 1C), indicating that certain

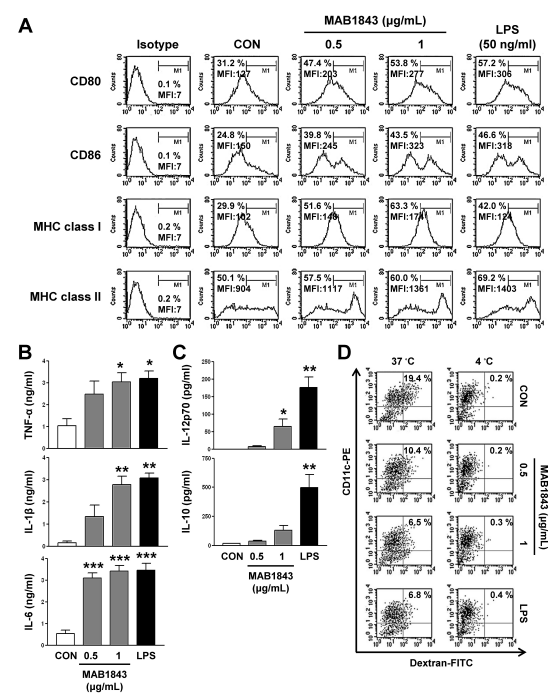

Fig. 2. MAB1843 induces the maturation of dendritic cells (DCs). (A) Immature DCs were cultured with 0.5 or $1 \mu \mathrm{g} / \mathrm{ml}$ of MAB1843 or $50 \mathrm{ng} / \mathrm{ml}$ of LPS for $24 \mathrm{~h}$ and analyzed for the expression of surface markers by two-color flow cytometry. DCs were stained with FITC anti-CD11c antibodies and PE anti-CD80 antibodies, PE anti-CD86 antibodies, PE anti-MHC class I antibodies, or PE anti-MHC class II antibodies. (B and C) Immature DCs were cultured with 0.5 or $1 \mu \mathrm{g} / \mathrm{ml}$ of MAB1843 or $50 \mathrm{ng} / \mathrm{ml}$ of LPS for $24 \mathrm{~h}$. The amounts of TNF- $\alpha$, IL-1 $\beta$, IL-6, IL-12p70, and IL-10 in the culture medium were measured by ELISA. The experiments were run at least in triplicates. ${ }^{*} P<0.05,{ }^{*} * \mathrm{P}<0.01$, and $* * * P<0.001$, when compared with DCs treated with MAB1843 only. (D) Mature DCs were generated by stimulating the immature cells with $50 \mathrm{ng} / \mathrm{ml}$ of LPS or 0.5 or $1 \mu \mathrm{g} / \mathrm{ml}$ of MAB1843 for 24 h. Endocytic activity at $37^{\circ} \mathrm{C}$ and at $4^{\circ} \mathrm{C}$ was assessed by flow cytometry, based on dextran-FITC uptake. The percentages of dextran-FITC ${ }^{+} \mathrm{CD}_{11 \mathrm{C}^{+}}$cells are indicated. The results are representative of three independent experiments. 
amounts of MAB1843 are not cytotoxic to DCs. Next, to test the physiological effect of MAB1843 on DC maturation, we analyzed the expression of surface molecules and the secretion of cytokines in MAB1843- or LPS-induced (positive control) DCs. MAB1843 enhanced the expression of surface molecules such as CD80, CD86, and MHC class I and II (Fig. $2 \mathrm{~A})$, and promoted the secretion of proinflammatory cytokines such as TNF- $\alpha$, IL-1 $\beta$, and IL-6 (Fig. 2B), but His-tagged protein itself did not affect to the production of cytokines in DCs (Supplementary Fig. 1). Whereas LPS stimulated the production of both IL-12p70 (involved in Th1 polarization) and IL-10 (involved in Th2 polarization), MAB1843 specifically induced the secretion of IL-12p70 only (Fig. 2C), indicating that it may promote Th1 polarization. Furthermore, MAB1843 suppressed the capacity of dextran and LPS to induce endocytosis in DCs, indicating that it can functionally provoke the maturation of DCs towards promoting Th1 polarization.

\section{MAB1843 Induces DC Maturation via TLR4 Signaling}

Toll-like receptors of immune cells, such as macrophages and DCs, act as initiators of innate immunity by recognizing various pathogen-associated molecular patterns (PAMPs) (23, 24). Apart from PAMPs, other $M$. abscessus antigens can activate DC maturation through $\operatorname{TLR} 2$ or $\operatorname{TLR} 4(7,8)$. Thus, we

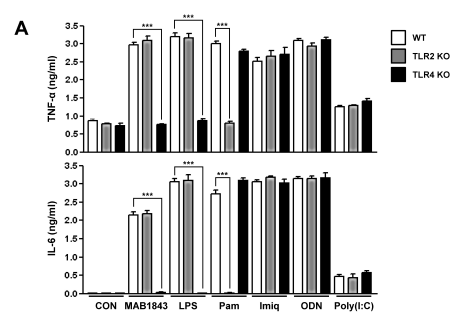

B

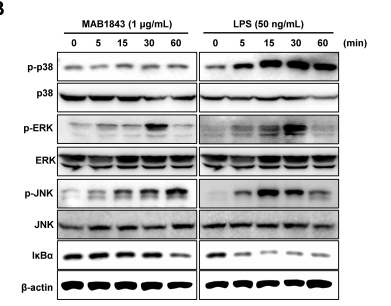

Fig. 3. MAB1843 induces TLR4 signaling during dendritic cell (DC) maturation. (A) DCs derived from wild type (WT), TLR2 knockout (KO), and TLR4 KO mice were treated with MAB1843 $(1 \mu \mathrm{g} / \mathrm{ml})$, LPS ${ }^{2}(50 \mathrm{ng} / \mathrm{ml})$, Pam3CSK4 (Pam3) (10 $\left.\mu \mathrm{g} / \mathrm{ml}\right)$, imiquimod (Imiq) $(1 \mu \mathrm{g} / \mathrm{ml})$, ODN1826 (ODN) $(1 \mu \mathrm{g} / \mathrm{ml})$, or Poly l:C $(10 \mu \mathrm{g} / \mathrm{ml})$ for $24 \mathrm{~h}$. The production of TNF- $\alpha$ and IL- 6 in DCs was measured by ELISA. The experiments were run at least in triplicates. $* * * P<$ 0.001. (B) DCs were treated with MAB1843 $(1 \mu \mathrm{g} / \mathrm{ml})$ or LPS (50 $\mathrm{ng} / \mathrm{ml}$ ) for $0,5,15,30$, and $60 \mathrm{~min}$. Anti-p-p38 antibody, antip-ERK antibody, anti-p-JNK antibody, anti-p38 antibody, anti-ERK antibody, anti-JNK antibody, anti-IKB $\alpha$ antibody, and anti- $\beta$-actin antibody was added to the western blot membrane. The results are representative of three independent experiments. tested whether MAB1843 can modulate DC maturation through TLRs as well. We analyzed the secretion of proinflammatory cytokines, such as TNF- $\alpha$ and IL-6, in wild type $(\mathrm{WT})$, TLR2 knockout $(\mathrm{KO})$, and TLR4 KO DCs treated with MAB1843, LPS (TLR4 agonist), Pam3CSK4 (TLR1/2 agonist), imiquimod (TLR7 agonist), ODN1826 (TLR9 agonist), or Poly I:C (TLR3 agonist). Whereas the secretion of TNF- $\alpha$ and IL-6 was observed in all three cell types stimulated by imiquimod, ODN1826, or Poly I:C, both cytokines were completely inhibited in Pam3CSK4-stimulated TLR2 KO DCs and in LPS-stimulated TLR4 KO DCs. The secretion of cytokines was completely blocked only in TLR4 KO DCs stimulated by MAB1843 or LPS (Fig. 3A), indicating that MAB1843 can modulate DC maturation through TLR4. As mitogen-activated protein kinases (MAPKs) and nuclear factor-kappa B (NF-kB) signaling are reportedly downstream mediators in TLR4 signaling (24), we investigated whether MAB1843 could enhance the activation of these two mediators. We measured the phosphorylation of MAPKs and the expression of NF- $\mathrm{KB}$

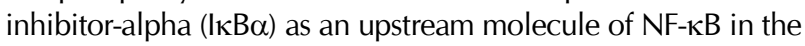
cytosol. MAB1843 promoted the activation of MAPKs and NF-кB in DCs derived from WT and TLR2-knockout mouse but not TLR4-knockout mouse (Fig. 3B and Supplementary Fig. 2). In addition, to investigate MAB1843 how to activate TLR4 signaling, we measured the interaction between TLR4 and MAB1843 using the BLitz system (Supplementary Fig. 3). These results indicate that MAB1843 could induce the DC activation occurs through the activation of TLR4-mediated MAPKs and NF- $\kappa B$ signaling by direct binding with TLR4.
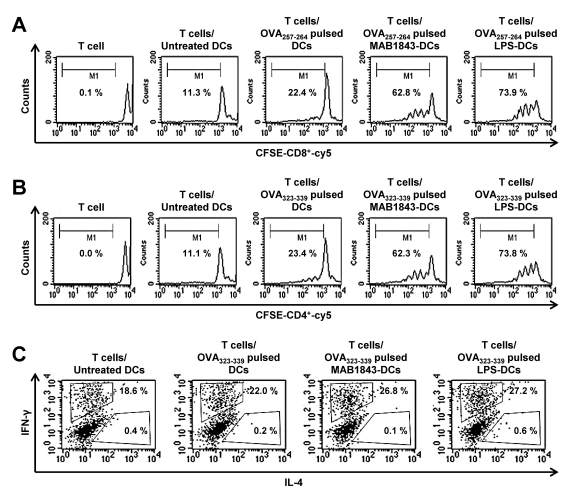

Fig. 4. MAB1843 induces $T$ cell proliferation and Th1 polarization. (A and B) Transgenic OVA-specific CD8 ${ }^{+} \mathrm{T}$ cells and transgenic OVAspecific $\mathrm{CD}^{+} \mathrm{T}$ cells were isolated from OT-I and OT-II mice, respectively. T cells were stained with carboxyfluorescein succinimidyl ester and co-cultured for $96 \mathrm{~h}$ with DCs treated with $1 \mu \mathrm{g} / \mathrm{ml}$ of MAB1843 or $50 \mathrm{ng} / \mathrm{ml}$ of LPS. The cells were then analyzed by flow cytometry. (C) Transgenic OVA-specific $\mathrm{CD}^{+} \mathrm{T}$ cells were co-cultured for 3 days with non-stimulated DCs, OVA $323-339-$ pulsed DCs, OVA $323-339$-pulsed MAB1843-DCs, or OVA $323-339$-pulsed LPStreated DCs. The $\mathrm{CD}^{+}{ }^{+} \mathrm{T}$ cell expression of IFN- $\gamma$ and IL-4 was detected by intracellular staining and flow cytometry. The results are representative of three independent experiments. 


\section{MAB1843 enhances $\mathrm{CD4}^{+}$and $\mathrm{CD8}^{+} \mathrm{T}^{-}$cell proliferation through DC maturation}

Activated DCs mature during their migration to the lymph nodes. Once mature, they present antigens to naïve T cells and induce $T$ cell proliferation (12). To characterize the effect of MAB1843 on $\mathrm{DC}$ and $\mathrm{T}$ cell interactions, we conducted a syngeneic mixed lymphocyte reaction assay using OT-I T cell receptor (TCR) transgenic $\mathrm{CD}^{+}{ }^{+} \mathrm{T}$ cells and OT-II TCR transgenic CD4 ${ }^{+} \mathrm{T}$ cells (25). The carboxyfluorescein succinimidyl ester (CFSE)-conjugated ovalbumin (OVA)-specific $\mathrm{CD} 8{ }^{+}$and $\mathrm{CD} 4{ }^{+} \mathrm{T}$ cells were divided and co-cultured with DCs presenting $\mathrm{OVA}_{257-264}$ (Fig. 4A) or $\mathrm{OVA}_{323-339}$ (Fig. 4B), which were effectively activated by MAB1843 treatment and LPS stimulation. In addition, MAB1843 also potentiated the production of IFN- $\gamma$ by the activated $\mathrm{CD}^{+}{ }^{+} \mathrm{T}$ cells, but not that of IL-4 (Fig. 4C). Considering these results and the fact that MAB1843 stimulated the DC production of IL-12p70 (Fig. 1C), we propose that this effect could be used in immunotherapy to polarize naïve $\mathrm{CD}^{+}$and $\mathrm{CD} 8^{+} \mathrm{T}$ cells towards an IFN- $\gamma$-producing Th1 phenotype.

\section{DISCUSSION}

Several strains of non-tuberculous mycobacteria, such as Mycobacterium xenopi and Mycobacterium kansasii, have been known to cause pulmonary diseases $(26,27)$. Recent research has shown that $M$. abscessus induces an immune response through TLR2 and dectin- 1 on host cells $(7,9)$, and MAB2560, the first purified antigen isolated from $M$. abscessus, activates DCs via TLR4 (8). It is essential to understand the relationship between $M$. abscessus and the immune system to develop a treatment strategy against infection by this species. Therefore, it was necessary to complete the research on how the immune system responds to M. abscessus-derived proteins. In this study, we investigated the effect of MAB1843, another purified antigen isolated from $M$. abscessus, on the immune system.

Here, we report that MAB1843 plays a role as an adjuvant to induce the secretion of proinflammatory cytokines and Th1 polarization via DC stimulation. Specifically, MAB1843 induced the maturation of DCs and the secretion of proinflammatory cytokines (viz., IL-12p70, IL-1 $\beta$, IL-6, and TNF- $\alpha$ ) via TLR4, both actions of which are important in T cell activation.

It has been reported that the MAPK and NF-KB pathways, activated by TLR signaling, are involved in the maturation of DCs and the secretion of proinflammatory cytokines $(28,29)$. In line with these studies, we found that MAB1843 induced the activation of MAPKs and NF-кB in DCs, which is important since DC maturation and proinflammatory cytokine production play a key role in T cell polarization. Therefore, it is possible that MAB1843 enhances the production of IL-12 in DCs through the activation of MAPKs and NF- $\mathrm{KB}$, which in turn elicits the differentiation of $\mathrm{CD}^{+}{ }^{+} \mathrm{T}$ cells into Th1 cells, which produce IFN- $\gamma$. In line with these suggestions, we found that MAB1843 does indeed elicit Th1 polarization and IFN- $\gamma$ production in Th1 cells. In this way, MAB1843 might be the link between the innate immune response by DCs and the adaptive immune response by Th1 cells to bacterial infection.

Our findings suggest that MAB1843 activates DCs and generates a Th1 response, as well as induces Th1 polarization by enhancing IL-12 production in DCs and boosting IFN- $\gamma$ production in $\mathrm{T}$ cells. However, further in vivo studies are necessary to completely verify the immune response to $M$. abscessus.

In conclusion, MAB1843 is a potent protein that induces DC maturation and Th1-mediated immune response. Knowledge on the mechanism of how MAB1843 modulates the activity of DCs could contribute to the development of vaccines against M. abscessus. The identification of mycobacterial antigens that could be used as adjuvants to induce the activation of APCs may direct the development of immunotherapy in the future.

\section{MATERIALS AND METHODS}

\section{Animals}

Female 4-6-week-old C57BL/6 $\left(\mathrm{H}-2 \mathrm{~K}^{\mathrm{b}}\right.$ and $\left.\mathrm{I}-\mathrm{A}^{\mathrm{b}}\right)$ mice were purchased from Orient Bio (Daejeon, South Korea). TLR2 and TLR4 KO mice, as well as transgenic OT-I and OT-II mice, were purchased from Jackson Laboratory (Bar Harbor, ME, USA). All of the animals were housed in a specific pathogenfree facility and treated in accordance with the animal care guidelines (Institutional Animal Care and Use Committee (IACUC) number: KU14046).

\section{Reagents and antibodies}

Recombinant mouse granulocyte macrophage colony stimulating factor (rmGM-CSF) was purchased from BioLegend (San Diego, CA, USA). The Annexin V/PI apoptosis detection kit was purchased from BD Biosciences (East Rutherford, NJ, USA). Fluorescein isothiocyanate (FITC)- or phycoerythrin (PE)-conjugated Abs-namely, FITC anti-mouse CD11c Abs (Clone: N418), PE anti-mouse CD80 Abs (Clone: 16-10A1), PE anti-mouse CD86 Abs (Clone: GL-1), PE anti-mouse $\mathrm{H}-2 \mathrm{Kd}$ / $\mathrm{H}-2 \mathrm{Dd}$ Abs (Clone: 34-1-2S), PE anti-mouse I-A/I-E Abs (Clone: M5/114.15.2), FITC anti-mouse IFN- $\gamma$ Abs (Clone: XMG1.2), and PE anti-mouse IL-4 Abs (Clone: BVD4-1D11)-were purchased from BioLegend. TNF- $\alpha$, IL-1 $\beta$, IL-6, IL-10, and IL-12p70 ELISA kits were purchased from eBioscience (San Diego, CA, USA). FITC-conjugated dextran was purchased from Sigma-Aldrich (St. Louis, MO, USA). The TLR agonists (viz., LPS from Escherichia coli O111:B4, Pam3CSK4, imiquimod, Poly I:C, and ODN1826) were purchased from Invivogen (San Diego, CA, USA). Antibodies against phosphoERK, phospho-p38, phospho-JNK, I $\mathrm{KB} \alpha$, and $\beta$-actin were purchased from Santa Cruz Biotechnology (Santa Cruz, CA, USA). CFSE was purchased from Life Technologies (Eugene, OR, USA). 


\section{Cloning of the MAB1843 gene}

The MAB1843 gene was isolated from the full genome of $M$. abscessus for cloning into the E. coli BL21 strain. MAB1843 was amplified using a forward primer (5'-CATATGATGAA GCGTCTGATCCTCGGT-3') and a reverse primer (5'-AAGC TTGTTGACGGGCGCGTTCAGGAA-3'). Thereafter, the PCR products were cleaved by Ndel and HindIII, and the cleaved genes were then inserted into the $\mathrm{pET}-22 \mathrm{~b}(+)$ vector (Novagen, Madison, WI, USA).

\section{Purification of MAB1843 from E. coli BL21}

The transformed E. coli BL21 was incubated in Luria-Bertani broth containing $100 \mu \mathrm{g} / \mathrm{ml}$ of ampicillin. When the optical density at $600 \mathrm{~nm}$ reached $0.4-0.6,1 \mathrm{mM}$ of isopropylthio- $\beta$ D-galactoside was added to the E. coli BL21 culture medium, which was then incubated for $6 \mathrm{~h}$. The $E$. coli BL21 cells were then harvested and lysed using lysozyme, dithiothreitol, phenylmethanesulfonyl fluoride (PMSF), and DNase I. The supernatant was analyzed by affinity chromatography with Ni-NTA agarose resin. The eluted solution was concentrated and dialyzed against phosphate-buffered saline (PBS). Finally, endotoxins were removed by polymyxin B-agarose (SigmaAldrich), and the endotoxin level was measured using the LAL test kit (Lonza).

\section{Generation of murine bone marrow dendritic cells}

DCs were collected from the bone marrow of the tibia and femora of C57BL/6 mice. The progenitor cells were isolated from the bone marrow, and red blood cell lysis buffer was added (Sigma-Aldrich). These cells were then mixed with RPMI 1640 medium containing 10\% fetal bovine serum, 1\% penicillin/streptomycin, and GM-CSF. The cells were seeded into 12-well cell culture plates and incubated at $37^{\circ} \mathrm{C}$ in a $5 \%$ $\mathrm{CO}_{2}$ atmosphere. Fresh medium was added on the third day. On the sixth day, the cells were further treated or harvested.

\section{Western blot analysis}

The harvested cells were washed with PBS and lysed using a lysis buffer (containing 0.5\% NP-40, $1 \mathrm{mM}$ EDTA, $50 \mathrm{mM}$ Tris- $\mathrm{HCl}$ (pH 8.0), $120 \mathrm{mM} \mathrm{NaCl}, 0.5 \mathrm{mM}$ PMSF, 0.5 M NaF, and $0.01 \%$ protease inhibitor cocktail (Biobasic, Amherst, NY, USA)). SDS-PAGE was conducted using a $10 \%$ polyacrylamide gel, following which the protein bands were transferred to a polyvinylidene difluoride membrane. After blocking the membrane with 5\% skimmed milk, primary Abs (anti-p-p38,

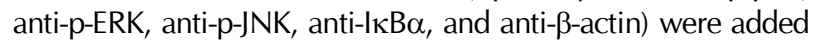
to the membrane and incubation was carried out overnight at $4^{\circ} \mathrm{C}$. On the next day, after rinsing with Tris-buffered saline and Tween 20, the anti-mouse and anti-rabbit secondary Abs were added to the membrane and a further incubation for $1 \mathrm{~h}$ at room temperature (RT) was carried out. After rinsing the membrane, enhanced chemiluminescence solution was added, and target bands were identified using the LAS-4000 imaging system (Fuji Film, Tokyo, Japan). To enable interaction with other antibodies, stripping buffer (Thermo Scientific, Waltham, MA, USA) was added to the membrane, following which the blocking step was repeated, and new antibodies were added.

\section{Cytotoxicity analysis}

MAB1843- or LPS-treated DCs were harvested and rinsed with PBS. Annexin V/PI staining was performed on these cells for $15 \mathrm{~min}$ at RT. The cytotoxicity of MAB1843 on DCs was analyzed by flow cytometry using the FACS Calibur instrument (BD Biosciences).

\section{Endocytosis ability analysis}

MAB1843- and LPS-treated DCs were treated with FITCconjugated dextran and incubated for $45 \mathrm{~min}$ at $37^{\circ} \mathrm{C}$ and $4^{\circ} \mathrm{C}$, respectively. The DCs were then harvested and rinsed with PBS. Thereafter, the DCs were stained using the PE-conjugated anti-CD11c Ab and analyzed by flow cytometry.

\section{Mixed Lymphocyte Reaction (MLR)}

MAB1843- or LPS-treated DCs were incubated with OVA $257-264$ or OVA $323-339$. Then, $1 \times 10^{4} \mathrm{DCs}$ and $1 \times 10^{5}$ CFSE-labeled T cells were co-cultured in a 96-well cell culture plate. These transgenic OVA-specific $\mathrm{CD}^{+}$and $\mathrm{CD}^{+} \mathrm{T}$ cells were isolated from splenocytes of OT-I and OT-II mice, respectively. After $96 \mathrm{~h}$, the cells were harvested and rinsed with PBS. Then, the cells were stained with Cy5 anti-CD8 or Cy5 anti-CD4 Abs and further analyzed by flow cytometry.

\section{Intracellular staining}

The harvested and rinsed cells were fixed in $4 \%$ paraformaldehyde and then permeabilized using saponin buffer. The cells were stained with anti-IFN- $\gamma$ and anti-IL-4 Abs for 20 min.

\section{Enzyme-Linked immunosorbent assay}

The levels of IL-12p70, IL-10, IL-1 $\beta$, TNF- $\alpha$, IL-6, and IFN- $\gamma$ were measured by ELISA (eBioscience; R\&D Systems, Minneapolis, MN, USA).

\section{Blitz assay}

The direct binding between rmTLR4/MD2 and MAB1843 was performed using the BLItz system (ForteBio, Menlo Park, CA). Human rTLR4/MD2 tagged with anti-penta-His (HIS) was purchased from R\&D Systems (Minneapolis, MN). HIS biosensors (catalog no. 18-5078, Fortebio) were hydrated for 10 min prior to the experiment. The concentration of HIS-tagged rmTLR4/MD2 was $0.1 \mathrm{mg} / \mathrm{ml}$, and that of purified MAB1843 was $0.1,0.5$, and $1 \mathrm{mg} / \mathrm{ml}$ and BSA was $1 \mathrm{mg} / \mathrm{ml}$. The setting was as follows: initial baseline for $30 \mathrm{~s}$, loading for $300 \mathrm{~s}$, baseline for $60 \mathrm{~s}$, association for $120 \mathrm{~s}$, and dissociation for $120 \mathrm{~s}$.

\section{Statistical analysis}

All experiments were repeated at least three times, with consistent results. Unless otherwise stated, data are expressed as the mean \pm SEM. Variance analysis was used to compare 
experimental groups with control values, whereas comparisons between multiple groups were made using Tukey's multiple comparison tests (Prism 3.0; GraphPad software). A P value of less than 0.05 was considered to indicate statistical significance.

\section{ACKNOWLEDGEMENTS}

This study was supported by a National Research Foundation (NRF) of Korea grant, funded by the Korean Government (NRF-2014R1A1A2054999 and NRF-2015R1A2A1A13001713), and the Basic Research Laboratory Program through NRF Korea, funded by the Ministry of Science, ICT \& Future Planning (NRF-2013R1A4A1069575).

\section{REFERENCES}

1. Rolston KV, Jones PG, Fainstein V and Bodey GP (1985) Pulmonary disease caused by rapidly growing mycobacteria in patients with cancer. Chest 87, 503-506

2. Set R and Shastri J (2011) Laboratory aspects of clinically significant rapidly growing mycobacteria. Indian J Med Microbiol 29, 343-352

3. Jeon K, Kwon OJ, Lee NY et al (2009) Antibiotic treatment of Mycobacterium abscessus lung disease: a retrospective analysis of 65 patients. Am J Respir Crit Care Med 180, 896-902

4. Lee MR, Sheng WH, Hung CC, Yu CJ, Lee LN and Hsueh PR (2015) Mycobacterium abscessus Complex Infections in Humans. Emerg Infect Dis 21, 1638-1646

5. Maurer FP, Bruderer VL, Ritter C, Castelberg C, Bloemberg GV and Bottger EC (2014) Lack of antimicrobial bactericidal activity in Mycobacterium abscessus. Antimicrob Agents Chemother 58, 3828-3836

6. Nessar R, Cambau E, Reyrat JM, Murray A and Gicquel B (2012) Mycobacterium abscessus: a new antibiotic nightmare. J Antimicrob Chemother 67, 810-818

7. Shin DM, Yang CS, Yuk JM et al (2008) Mycobacterium abscessus activates the macrophage innate immune response via a physical and functional interaction between TLR2 and dectin-1. Cell Microbiol 10, 1608-1621

8. Lee SJ, Shin SJ, Lee SJ et al (2014) Mycobacterium abscessus MAB2560 induces maturation of dendritic cells via Toll-like receptor 4 and drives Th1 immune response. BMB Rep 47, 512-517

9. Sampaio EP, Elloumi HZ, Zelazny A et al (2008) Mycobacterium abscessus and $M$. avium trigger Toll-like receptor 2 and distinct cytokine response in human cells. Am J Respir Cell Mol Biol 39, 431-439

10. Haverkamp MH, van Dissel JT and Holland SM (2006) Human host genetic factors in nontuberculous mycobacterial infection: lessons from single gene disorders affecting innate and adaptive immunity and lessons from molecular defects in interferon-gamma-dependent signaling. Microbes Infect 8, 1157-1166

11. Rottman M, Catherinot E, Hochedez $P$ et al (2007) Importance of T cells, gamma interferon, and tumor necrosis factor in immune control of the rapid grower Mycobacterium abscessus in C57BL/6 mice. Infect Immun 75, 5898-5907
12. Banchereau J and Steinman RM (1998) Dendritic cells and the control of immunity. Nature 392, 245-252

13. Banchereau J and Palucka AK (2005) Dendritic cells as therapeutic vaccines against cancer. Nat Rev Immunol 5, 296-306

14. Palucka K and Banchereau J (2012) Cancer immunotherapy via dendritic cells. Nat Rev Cancer 12, 265-277

15. Schuler G, Schuler-Thurner B and Steinman RM (2003) The use of dendritic cells in cancer immunotherapy. Curr Opin Immunol 15, 138-147

16. Bansal K, Elluru SR, Narayana $Y$ et al (2010) PE_PGRS antigens of Mycobacterium tuberculosis induce maturation and activation of human dendritic cells. J Immunol 184, 3495-3504

17. Pecora ND, Gehring AJ, Canaday DH, Boom WH and Harding CV (2006) Mycobacterium tuberculosis LprA is a lipoprotein agonist of TLR2 that regulates innate immunity and APC function. J Immunol 177, 422-429

18. Heo DR, Shin SJ, Kim WS et al (2011) Mycobacterium tuberculosislpdC, Rv0462, induces dendritic cell maturation and Th1 polarization. Biochem Biophys Res Commun 411, 642-647

19. Lee SJ, Noh KT, Kang TH et al (2014) The Mycobacterium avium subsp. Paratuberculosis protein MAP1305 modulates dendritic cell-mediated T cell proliferation through Toll-like receptor-4. BMB Rep 47, 115-120

20. Jung ID, Jeong SK, Lee CM et al (2011) Enhanced efficacy of therapeutic cancer vaccines produced by co-treatment with Mycobacterium tuberculosis heparin-binding hemagglutinin, a novel TLR4 agonist. Cancer Res 71, 2858-2870

21. Jung ID, Shin SJ, Lee MG et al (2014) Enhancement of tumor-specific $\mathrm{T}$ cell-mediated immunity in dendritic cell-based vaccines by Mycobacterium tuberculosis heat shock protein X. J Immunol 193, 1233-1245

22. Lee SJ, Shin SJ, Lee MH et al (2014) A potential protein adjuvant derived from Mycobacterium tuberculosis Rv0652 enhances dendritic cells-based tumor immunotherapy. PLoS One 9, e104351

23. O'Neill LA, Golenbock D and Bowie AG (2013) The history of Toll-like receptors - redefining innate immunity. Nat Rev Immunol 13, 453-460

24. van Duin D, Medzhitov R and Shaw AC (2006) Triggering TLR signaling in vaccination. Trends Immunol 27, 49-55

25. Hogquist KA, Jameson SC, Heath WR, Howard JL, Bevan MJ and Carbone FR (1994) T cell receptor antagonist peptides induce positive selection. Cell 76, 17-27

26. This official statement of the American Thoracic Society was approved by the Board of Directors (1997) Diagnosis and treatment of disease caused by nontuberculous mycobacteria. Am J Respir Crit Care Med 156, S1-25

27. Marras TK and Daley CL (2002) Epidemiology of human pulmonary infection with nontuberculous mycobacteria. Clin Chest Med 23, 553-567

28. Hayashi F, Smith KD, Ozinsky A et al (2001) The innate immune response to bacterial flagellin is mediated by Toll-like receptor 5. Nature 410, 1099-1103

29. Lee JS, Jung ID, Jeong YI et al (2007) D-pinitol inhibits Th1 polarization via the suppression of dendritic cells. Int Immunopharmacol 7, 791-804 